\title{
Prostate specific membrane antigen-radio guided surgery using Cerenkov luminescence imaging-utilization of a short-pass filter to reduce technical pitfalls
}

\author{
Christopher Darr ${ }^{1,2}$, Pedro Fragoso Costa ${ }^{2,3}$, Claudia Kesch ${ }^{1,2}$, Ulrich Krafft ${ }^{1,2}$, Lukas Püllen ${ }^{1,2}$, \\ Nina Natascha Harke ${ }^{1,2}$, Jochen Hess ${ }^{1,2}$, Tibor Szarvas ${ }^{1,2}$, Johannes Haubold ${ }^{2,4}$, Henning Reis ${ }^{2,5}$, \\ Wolfgang Peter Fendler, ${ }^{2,3}$, Ken Herrmann ${ }^{2,3}$, Jan Philipp Radtke ${ }^{1,2}$, Boris Alexander Hadaschik ${ }^{1,2 \#}$, \\ Stephan Tschirdewahn ${ }^{1,2 \#}$
}

${ }^{1}$ Department of Urology, University Hospital Essen, Essen, Germany; ${ }^{2}$ German Cancer Consortium (DKTK)-University Hospital Essen, Essen, Germany; ${ }^{3}$ Department of Nuclear Medicine, University Hospital Essen, Essen, Germany; ${ }^{4}$ Institute of Diagnostics and Radiology, University Hospital Essen, Essen, Germany; ${ }^{5}$ Institute of Pathology, University of Duisburg-Essen, Essen, Germany

Contributions: (I) Conception and design: BA Hadaschik, K Herrmann, JP Radtke, WP Fendler, C Darr; (II) Administrative support: J Hess, T Szarvas, J Haubold, H Reis; (III) Provision of study materials or patients: L Püllen, C Kesch, U Krafft, NN Harke; (IV) Collection and assembly of data: C Darr, P Fragoso Costa, S Tschirdewahn, J Haubold, H Reis; (V) Data analysis and interpretation: C Darr, BA Hadaschik, J Hess, T Szarvas; (VI) Manuscript writing: All authors; (VII) Final approval of manuscript: All authors.

\#These authors contributed equally to this work.

Correspondence to: Dr. med. Christopher Darr. Resident Urologist, Department of Urology, University Hospital Essen, Hufelandstr. 55, 45147 Essen, Germany. Email: christopher.darr@uk-essen.de.

Background: Intraoperative Cerenkov luminescence imaging (CLI) is a novel technique to assess surgical margins in patients undergoing nerve sparing radical prostatectomy (RP). Here, we analyze the efficacy of a 550-nm optical short-pass filter (OF) to improve its performance.

Methods: In this prospective single-center feasibility study ten patients with prostate cancer (PC) were included between December 2019 and April 2020, including three patients without tracer injection as a control group. After preoperative injection of 68-Ga-prostate-specific membrane antigen (PSMA)-11 followed by RP, CLI of the excised prostate and the incised index lesion was performed to visualize the primary tumor lesion. We compared the findings on intraoperative CLI to postoperative histopathology. Furthermore, CLI-intensities determined as tumor to background ratio (TBR) and contrast to noise ratio (CNR) were measured.

Results: Histopathology proved positive surgical margins (PSM) in 3 patients with corresponding findings in CLI. After magnetic resonance imaging (MRI)-informed incision above the index lesion 2 out of 3 prostates demonstrated elevated CLI signals with histopathological confirmation of PC cells. The use of the OF enabled a significant reduction of the area of the regions of interest from a median of 1.80 to $0.15 \mathrm{~cm}^{2}$ (reduction by $85 \%, \mathrm{P}=0.005$ ) leading to increased specificity. Signals due to PSMs were not suppressed by the 550-nm OF. The median TBR was reduced from 3.33 to 2.10. In all three patients of the control group elevated CLI intensities were detected at locations with diathermal energy deposition during surgery. After application of the 550-nm OF these were almost totally suppressed with a TBR of 1.10. Measurements of Cerenkov luminescence intensity with the 550-nm OF showed a significant Pearson's correlation of 0.82 between PSM and the elevated TBR $(\mathrm{P}=0.003)$ and a significant Pearson's correlation of 0.66 between PSM and elevated $\mathrm{CNR}(\mathrm{P}=0.04)$. Measurements without the $\mathrm{OF}$ did not correlate significantly.

Conclusions: Intraoperative 68-Ga-PSMA CLI in PC is a tool that warrants further investigation to visualize PSM especially in intermediate and high-risk PC. Intraoperative CLI benefits from usage of a 550-nm OF to reduce false-positive signals. 
Keywords: Prostate cancer (PC); radical prostatectomy (RP); Cerenkov luminescence imaging (CLI); margin assessment; 68-Ga-PSMA-11

Submitted Jul 31, 2020. Accepted for publication Dec 07, 2020.

doi: $10.21037 /$ tau-20-1141

View this article at: http://dx.doi.org/10.21037/tau-20-1141

\section{Introduction}

Radical prostatectomy (RP) is one of the primary treatment options for men with localized or locally advanced prostate cancer (PC) and aims at complete tumor resection without positive surgical margins (PSM) (1). Preoperative magnetic resonance imaging (MRI) improves knowledge of the localization of PC before RP (2,3). However, Gandaglia et al. recently demonstrated that both MRI and prostate sampling using systematic biopsy cores are needed to predict adverse pathology accurately (4). This is of relevance when planning patient-tailored preservation of the neurovascular bundles without increased risk and numbers of PSM $(5,6)$. In addition to preoperative MRI for local staging and nomograms for the prediction of extraprostatic extension, the use of intraoperative frozen section analysis (IFS) can help the surgeon to preserve functional structures like the neurovascular bundles (1,6-8). However, besides resource consumption, there is also some conflicting evidence, as studies demonstrated high false-negative rates of IFS, potentially resulting in unjustified nerve-sparing surgery $(7,9)$. Various pre- and intraoperative methods are currently part of scientific research with the aim of reducing or avoiding PSM as incomplete removal of aggressive cancer tissue during RP is associated with poorer patient outcomes (10).

Molecular imaging with modern radiopharmaceuticals that target the prostate-specific membrane antigen (PSMA) can be used for highly specific oncological diagnostic imaging, including initial staging of PC (11-14). PSMA positron emission tomography (PSMA-PET) is an imaging technique that targets PSMA on PC cells, mostly with ${ }^{68} \mathrm{Ga}-$ labelled and ${ }^{18} \mathrm{~F}$-labelled PET agents (15). In men with biochemical recurrence, PSMA-PET imaging has become the imaging modality of choice if the results will influence subsequent treatment decisions (16).

In recent trials, PSMA-PET demonstrated promising detection rates of lymph node metastases and high positive predictive values for initial staging of PC (17-20). A recently published randomized multicentre study proved a lower sensitivity and specificity for conventional primary staging with computed tomography (CT) and bone scan as compared to 68-Ga-PSMA/PET/CT (21). Maurer et al. described the advantage of PSMA-radio guided pelvic lymph node dissection, visualising even small lymph node metastasis (22). Consequently, there is a wish to be able to accurately detect malignant areas in real-time during RP to ensure that all PC is completely removed.

PET imaging agents also emit optical photons via a phenomenon called Cerenkov luminescence, enabling optical molecular imaging in the form of Cerenkov luminescence imaging (CLI) (23). Both techniquesCLI and PET-measure photons: PET measures gamma annihilation photons, and CLI measures Cerenkov photons (24). Cerenkov photons are emitted by a charged particle (positron or electron) when travelling through a dielectric medium at a faster speed than the velocity of light in that medium. Although Cerenkov luminescence has a broad wavelength spectrum, it predominantly comprises ultraviolet and blue light. These short wavelengths are highly attenuated in biological tissue. Therefore, CLI is limited to detection of signals emitted in superficial tissue layers (approximately $3 \mathrm{~mm}$ ). In contrast to PET, CLI is unable to detect photons emitted by deeper located tissues or tumors $(25,26)$. Intraoperative imaging with CLI is promising since it allows to evaluate the entire surface of the prostate instead of only specific IFS slices $(27,28)$. Besides the good agreement between CLI and histopathology, there is also evidence of false-positive signals mainly at the bladder neck (27). Artificial signals caused by longer wavelengths can make intraoperative assessment more difficult and even lead to unnecessary resections of healthy tissue.

With regard to CLI, only few studies with small patient populations have been published so far. To our knowledge there is no published data available regarding CLI in PC investigating the benefit of an additional optical short-pass filter (OF). The main purpose of the present feasibility study was to improve the CLI efficiency through a $550-\mathrm{nm}$ OF in order to filter out potential interfering signals and thus to improve detection of signals caused by 
carcinoma cells on the surface. The secondary objective was evaluation of potential interfering factors in relation to the intraoperative application of CLI and the different ratios for CLI assessment: Tumor to background ratio (TBR) and contrast to noise ratio (CNR). We present the following article in accordance with the STROBE reporting checklist (available at http://dx.doi.org/10.21037/tau-20-1141).

\section{Methods}

\section{Patient recruitment and patient preparation on day of surgery}

Patients for primary treatment with histologically confirmed intermediate and high-risk PC on systematic and/or MRItargeted prostate biopsy were recruited at one study center between December 2019 and April 2020 after discussion in the local multidisciplinary tumor board. The study was conducted in accordance with the Declaration of Helsinki (as revised in 2013) and after written informed consent of the patients. The study was approved by the institutional ethics board of the University Duisburg-Essen (approval number 19-8749-BO). The study has been registered at German Clinical Trials register (DRKS-ID: DRKS00020942) and is recruiting patients at the time of submission. Only patients with intraoperative incision of the prostate gland above the index lesion were included. Exclusion criteria were previous prostate surgery or radiotherapy, known distant metastases in conventional staging according to the actual European Association of Urology (EAU) guidelines, and contraindications to surgery (1).

On the day of surgery, 68-Ga-PSMA-11 (1.8-2.2 MBq per $\mathrm{kg} /$ body weight) was injected intravenously in patients scheduled to undergo RP (29). Forty-five to 60 minutes after tracer injection, a PET/CT scan was performed and assessed by experienced nuclear medicine physicians. In case of high-volume metastatic disease on PET/CT, same-day surgery would have been cancelled.

\section{$R P$ and intraoperative $C L I$}

Seven patients with preoperative 68-Ga-PSMA administration, intraoperative CLI as well as incision of the index lesion were included in the present feasibility study. The localization of the index lesion in the prostate was based on preoperative MRI and MRI-targeted biopsy. Final RP histopathology proved the accuracy of the localisation. After PET/CT, RP was performed by two surgeons ahead of extended pelvic lymph node dissection (ePLND) to minimize signal intensity (SI) reduction from radiotracer decay in the time between 68-Ga-PSMA injection and CLI. A urinary catheter was inserted in the operating room (OR) to drain the urine, minimizing urinary contamination of the prostate. The excised prostate specimen was immediately retrieved from the abdomen, wiped to clear blood and fluids, positioned in a specimen tray and then imaged in the LightPath ${ }^{\circledR}$ CLI system (Lightpoint Medical Ltd., Chesham, UK). The LightPath ${ }^{\circledR}$ system captured both a Cerenkov image and a photographic image of the specimen in a light-tight imaging chamber and was positioned in the OR to enable real-time image analysis by the surgeon. Images were acquired with a total acquisition time of 300 seconds and $8 \times 8$ pixel binning (30). Each examination was repeated with a $550-\mathrm{nm}$ OF to ensure that the specimen was in the identical position. The prostate gland was imaged in different positions, and a total of two or three images were necessary to capture the entire surface area of the prostate. After the intact prostate was examined, the prostate gland was incised in the area of the index lesion on MRI and subsequently imaged in CLI to visualise the primary tumor. The incised specimens are imaged without the $550-\mathrm{nm}$ OF. Upon CLI completion, the RP specimen was sent for postoperative histopathological examination. Due to the design of this feasibility study and limited evidence on the performance characteristics of CLI, the surgical course remained unaffected by the CLI results and no further tissue was resected if positive margins were suspected on CLI.

\section{Image analysis}

The CLI images were analysed postoperatively in a controlled and standardized analysis environment using PMOD (PMOD Version 3.204, PMOD Technologies LLC). Measurements of the mean radiance (photons $/ \mathrm{s} / \mathrm{cm}^{2} / \mathrm{sr}$ ) were performed by manually drawing regions of interest (ROIs) on the CLI. ROIs were selected in areas showing increased SI and in areas with no increased SI (tissue background). Furthermore, TBRs as well as CNRs of the mean values were calculated. The following formulas have been used for the respective calculation: $\mathrm{TBR}=$ mean signal $/$ mean background, $\mathrm{CNR}$ $=($ mean signal - mean background $) /$ background standard deviation (31). Tumor margin assessment on CLI was performed by analysing elevated signals on the surface of the intact prostate. For background measurements a 
standardized ROI with an area of $0.8 \mathrm{~cm}^{2}$ was used. This standardized ROI was applied to each CLI image examined in this study. The area of the ROIs with elevated CLI intensities was measured with and without application of a $550-\mathrm{nm}$ OF and is stated in $\mathrm{cm}^{2}$. In case of missing CLI signals with the $550-\mathrm{nm}$ OF, the ROI of the measurement without $550-\mathrm{nm}$ OF was transposed and used to determine the CLI intensity. In this case the measured area was not statistically evaluated. It should also be noted that the background signal of the prostate is inhomogeneous. Thus, in the calculated ratios, some negative results can occur if the signal of one ROI is lower than that of the predefined background. False positive signals have been defined as a TBR $>1$ without histopathologic prove of cancer cells in that area.

\section{Histopathology}

Histopathologic evaluation was performed adherent to the current German S3-guidelines for PC (32). RPs were evaluated by an expert uro-pathologists with 12 years of experience in genitourinary-pathology (H Reis) and typical PC metrics were reported including Gleason grading in its 2014 revision (33). Quarters have been digitally reconstructed to whole mounts to be able to compare them with the CLI images.

\section{Statistical analysis}

Numerical variables were summarized with median values and interquartile ranges (IQRs), categorical variables with proportions (\%). To compare medians of non-parametric data, the Mann-Whitney $U$ test was used for two groups. Pearson's correlation coefficient was used to correlate PSM with elevated CLI intensities (significance set at $\mathrm{P}<0.05$ ). Statistical analysis was performed with IBM SPSS ${ }^{\circledR}$ Statistics version 26 (IBM, Armonk, USA).

\section{Results}

Patients' demographic and histopathological data are given in Table 1. Histopathology proved PSM in three patients with corresponding findings in CLI. Of note, in the seven patients undergoing 68-Ga-PSMA/PET-CT before surgery PET-avidity and multiparametric MRI (mpMRI) correlated with regards to the index lesion.

After MRI-informed incision above the index lesion 2 out of the 3 prostates with PSM demonstrated elevated
CLI signals in the index lesions (Figure 1). The anatomical correlation of the three patients with PSM is shown in Figure 2. Here the histopathological finding with a positive resection margin is shown with the corresponding CLI image. One patient had a TBR of 2.1 after application of the $550-\mathrm{nm}$ OF with a decay corrected activity level of $1.3 \mathrm{kBq} / \mathrm{mL}$ at the time of CLI analysis with an Internal Society of Urologic Pathology (ISUP) grade group of 4 (Figure 1, A1, B1, C1; Figure 2, images 1.1). In addition, this patient showed another suspicious CLI signal on the dorsal right surface of the prostate. A further positive resection margin was histopathologically confirmed here (Figure 2, images 1.2). The second patient with PSM but negative CLI findings of the incised lesion showed a TBR of 1.5 after application of the $550-\mathrm{nm}$ OF with a decay corrected activity level of $0.89 \mathrm{kBq} / \mathrm{mL}$ at the time of CLI analysis with an ISUP grade group of 2 (Figure 1, A2, B2, C2; Figure 2, images 2). The third patient with PSM and CLI signal showed a TBR of 2.15 after application of the 550$\mathrm{nm}$ OF with a decay corrected activity level of $0.34 \mathrm{kBq} / \mathrm{mL}$ at the time of CLI analysis with an ISUP grade group of 2 (Figure 2, images 3).

Activity levels at CLI varied according to different periods between tracer injection and CLI with a median of $0.38 \mathrm{kBq} / \mathrm{mL}(0.34 ; 1.3 \mathrm{kBq} / \mathrm{mL})$. Importantly, all of the three men within the control group showed CLI signals, especially at the bladder neck and in areas with prior use of electrocautery (Figure 3). In all ten cases the addition of the $550-\mathrm{nm}$ OF significantly reduced the area of displayed ROIs by $85 \%$ from a median of 1.8 to $0.15 \mathrm{~cm}^{2}$ (Table 2, $\mathrm{P}=0.005$ ). Noteworthy, only false positive signals disappeared. Positive signals in CLI caused by PSM have not been affected by the application of the $550-\mathrm{nm}$ OF. According to the histopathological results, three patients showed positive resection margins. All these patients had received prior tracer injection and all PSM were located at CLI suspicious areas and could be detected correctly with CLI. The median SI of the prostate background of the study group without $550-\mathrm{nm}$ OF was 2,392.00 photons/s/ $\mathrm{cm}^{2} / \mathrm{sr}$ (IQR, 1,849.17; 6,150.75 photons $/ \mathrm{s} / \mathrm{cm}^{2} / \mathrm{sr}$ ). The median SI using the 550-nm OF was significantly reduced to $1,465.50$ photons $/ \mathrm{s} / \mathrm{cm}^{2} / \mathrm{sr}$ (IQR, 1,373.00; $1,776.08$ photons $\left./ \mathrm{s} / \mathrm{cm}^{2} / \mathrm{sr}\right)(\mathrm{P}=0.018)$. The ratios, TBR and $\mathrm{CNR}$, were not significantly changed by the optical filter (Table 3).

Four patients with negative resection margins showed no noticeable Cerenkov luminescence in the incised index lesion. Representative images are shown in Figure 1, A3, B3, C3. Decay corrected activity levels for the time of CLI 
Table 1 Patient demographics, imaging and histopathologic characteristics

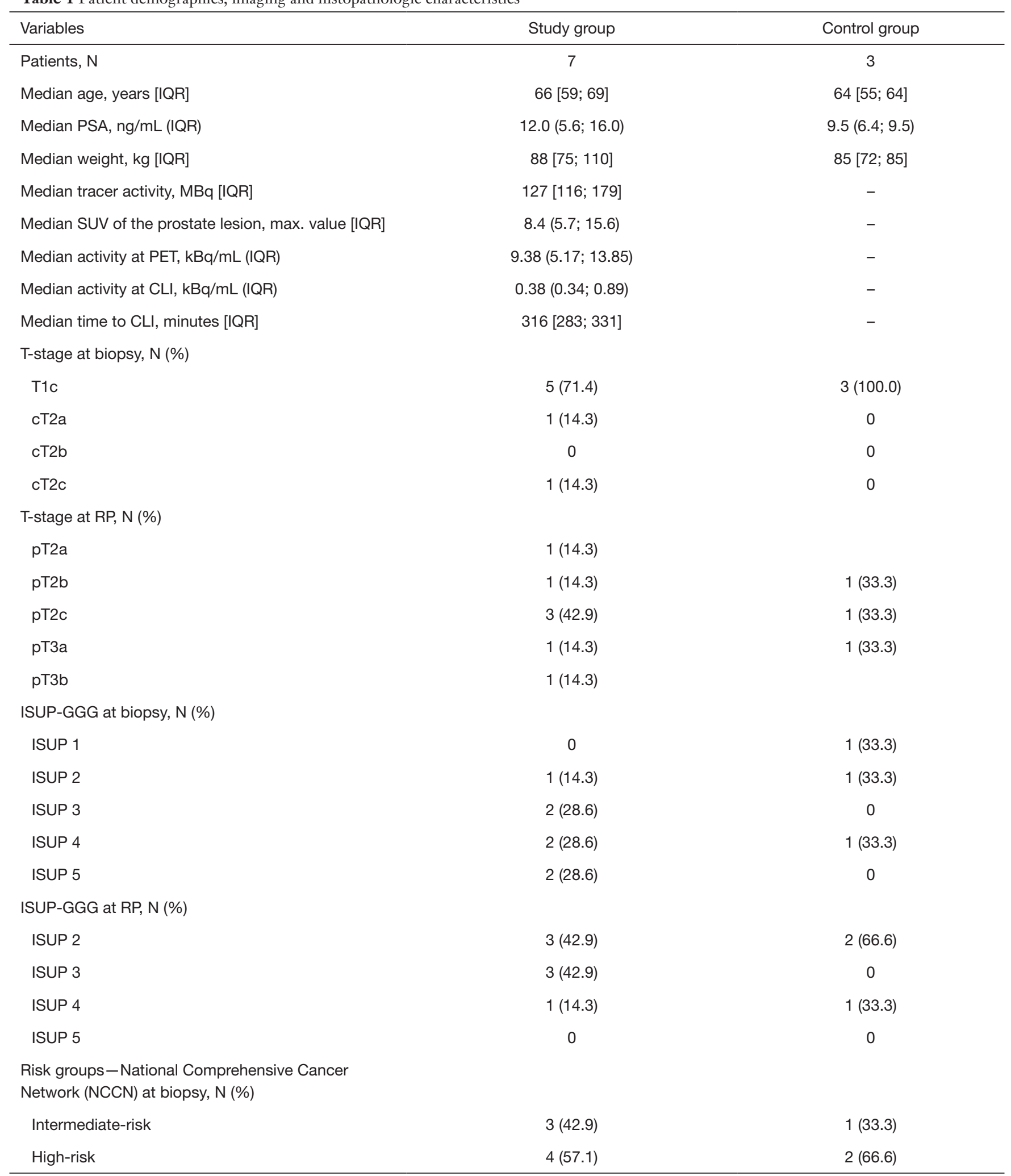

Table 1 (continued) 
Table 1 (continued)

\begin{tabular}{|c|c|c|}
\hline Variables & Study group & Control group \\
\hline \multicolumn{3}{|c|}{$\begin{array}{l}\text { Risk groups-National Comprehensive Cancer } \\
\text { Network (NCCN) at RP, N (\%) }\end{array}$} \\
\hline Intermediate-risk & $5(71.4)$ & $1(33.3)$ \\
\hline High-risk & $2(28.6)$ & $2(66.6)$ \\
\hline $\mathrm{R} 1, \mathrm{~N}(\%)$ & $3(42.9)$ & 0 \\
\hline $\mathrm{R} 1$ in $\mathrm{pT} 2, \mathrm{~N}$ (\% of all pT2) & $2(40.0)$ & 0 \\
\hline $\mathrm{R} 1$ in $\mathrm{pT} 3, \mathrm{~N}$ (\% of all pT3) & $1(50.0)$ & 0 \\
\hline
\end{tabular}

IQR, interquartile ranges; PSA, prostate specific antigen; SUV, standardized uptake value; PET, positron emission tomography; CLI, Cerenkov luminescence imaging; RP, radical prostatectomy; ISUP, International Society of Urological Pathology; GGG, Gleason grade groups; R0, negative surgical margin; R1, positive surgical margin.

investigation ranged from 0.33 to $0.78 \mathrm{kBq} / \mathrm{mL}$. ISUP grade group of these patients ranged from 2 to 3 .

Measurements of the CLI intensity with the 550-nm OF showed a significant Pearson's correlation of 0.82 between PSM and the elevated TBR $(\mathrm{P}=0.003$, Table 3). Measurements without the OF did not correlate significantly. Besides the TBR, the CNR was calculated, also demonstrating a significant Pearson's correlation of 0.66 $(\mathrm{P}=0.04)$ (Tables 2,3).

The examination of the two groups-patients with preoperative 68-Ga-PSMA and without tracer injectionshowed no significant differences between the examined TBR and CNR values if measured without OF highlighting the potential for false-positive CLI signals. After application of the OF a significant decrease of the TBR $(\mathrm{P}=0.02)$ was observed (Figure 3).

\section{Discussion}

This feasibility study evaluates the efficacy of intraoperative 68-Ga-PSMA CLI with an additional 550-nm OF for assessing tumor margin status in patients with primary PC. In general, CLI is able to detect PC cells close to the surface of the prostate gland as well as in lymph nodes $(27,28,34)$. Using the additional filter did not affect the sensitivity for detection of PSM in PC. Positive findings by CLI were possible down to a minimal activity of $0.34 \mathrm{kBq} / \mathrm{mL}$ at the time of examination.

In all ten examined prostates increased CLI intensities could be detected without the 550-nm OF. As already described in a previous publication, these intensities are frequently found at the bladder neck. A moderate to strong PSMA-staining in benign tissues may cause these CLI signals (27). After investigating the same prostate alignment with an additional $550-\mathrm{nm}$ OF, these signals were significantly reduced. To further investigate the origin of increased activity at non-cancerous surfaces, three patients undergoing RP without prior tracer injection were examined as a control group using CLI. After intraoperative prostate retrieval, the same procedure was performed as in patients with preoperative tracer injection. Interestingly, increased CLI intensities, mainly at the bladder neck, were also detected in these specimens, suggesting that this false positive, artificial signal is independent of tracer injection. Since the bladder neck, in contrast to the apex, is almost exclusively resected by electrocoagulation, it is reasonable to assume that the thermal alteration of the tissue generates radiation which is detected by the used system. Macroscopic examination of the suspect areas showed a strong correlation to areas of coagulated tissue. This was the case for both monopolar and bipolar devices. Accordingly, electrocoagulation was used sparsely in one patient, with a correspondingly lower occurrence of CLI signals. Importantly, here, we are able to demonstrate that these non-specific signals can be significantly reduced by using a 550-nm OF and therefore are assigned to a longer wavelength than the CLI signals of 68-Ga-PSMA-11.

68-Ga-PSMA-11 is known to be excreted via the urinary tract, and thus could also cause elevated signals at the bladder neck or prostate base through urinary 

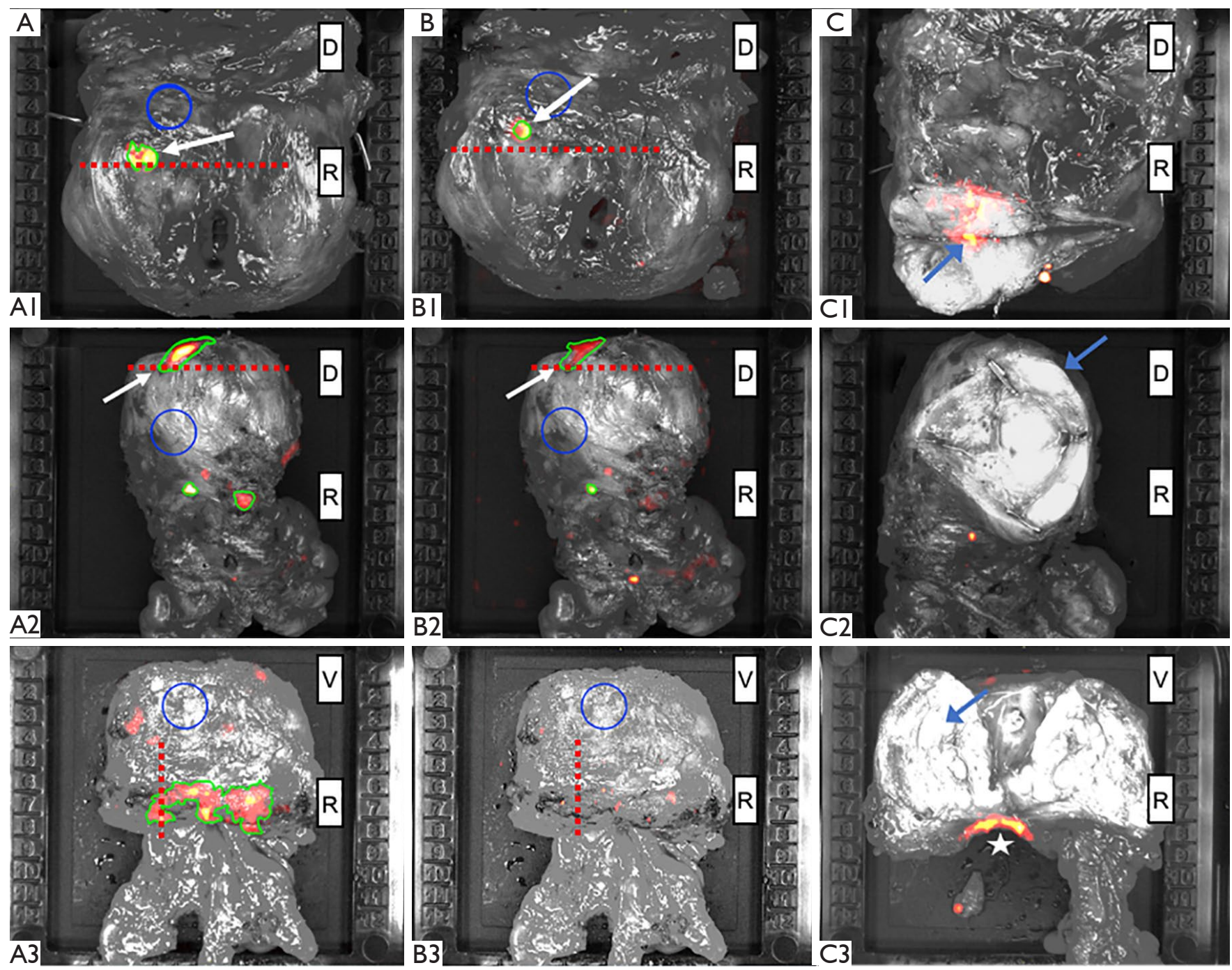

Figure 1 Grey-scale photographic images overlaid with Cerenkov signals, without the 550-nm optical short-pass filter (A) and with the 550-nm optical short-pass filter (B). The incised prostate above the index lesion is displayed in (C). The location of the incision is marked by a red dotted line on (A,B). The blue circles show the background ROIs. The suspicious CLI-intensity is labelled through green ROIs corresponding to the illuminating surface. (A1,B1,C1) Prostatectomy specimen with a positive resection margin as well as CLI-positivity of the incised lesion A high CLI-intensity at the left dorso-apical prostate is seen in both images without (TBR: 3.33, CNR: 16.2) and with (TBR: 2.1, CNR: 6.54) 550-nm optical short-pass filter and represented by an arrow. The incised specimen revealed a positive CLI signal corresponding with prostate cancer cells at this area (blue arrow). (A2,B2,C2) Prostatectomy specimen with a positive resection margin but CLI-negativity of the incised lesion A high CLI-intensity at the left apex of the prostate is seen in both images without (TBR: 2.79, CNR: 11.73) and with (TBR: 1.5, CNR: 2.44) 550-nm optical short-pass filter and represented by an arrow. The incised specimen revealed a negative CLI signal despite histopathological confirmation of prostate cancer cells at the incised area (blue arrow). (A3,B3,C3) One patient with negative resection margins and CLI-negativity of the incised lesion A high CLI-intensity at the bladder neck is seen (TBR: 3.36, CNR: 11.44), correlating with the usage of diathermy intraoperatively. After application of the 550-nm optical short-pass filter these signals are close the background intensity level (TBR: 1.1, CNR: 0.54). The incised specimen revealed a negative CLI signal despite histopathological confirmation of prostate cancer cells at the incised area (blue arrow). The star marks again an area of high CLI-intensity caused by diathermy since the specimen are imaged without the 550-nm optical short-pass filter. D, dorsal; R, right side; V, ventral; ROIs, regions of interest; CLI, Cerenkov luminescence imaging; TBR, tumor to background ratio; CNR, contrast to noise ratio. 

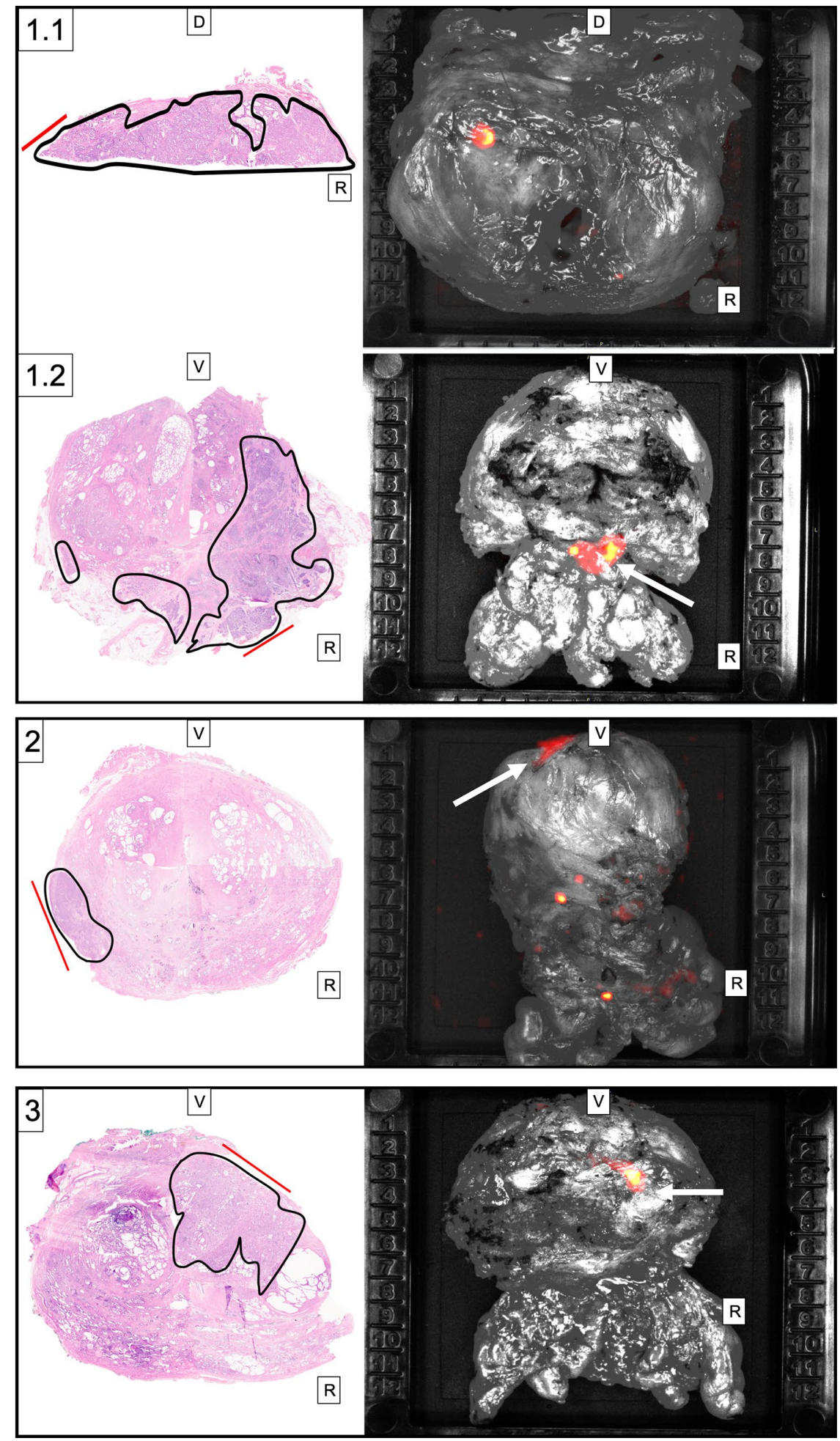

Figure 2 Correlation of the histopathological positive surgical margin (on the left) with the corresponding CLI image (on the right). For this purpose, the quarters have been combined into a whole-mount where applicable. In the histopathological image, the prostate carcinoma tissue is outlined in black and the R1 status is shown with a red line. In the CLI image, the corresponding signal is marked with an arrow. All CLI images were generated with the 550-nm optical filter. CLI, Cerenkov luminescence imaging; R, right; D, dorsal; V, ventral. 

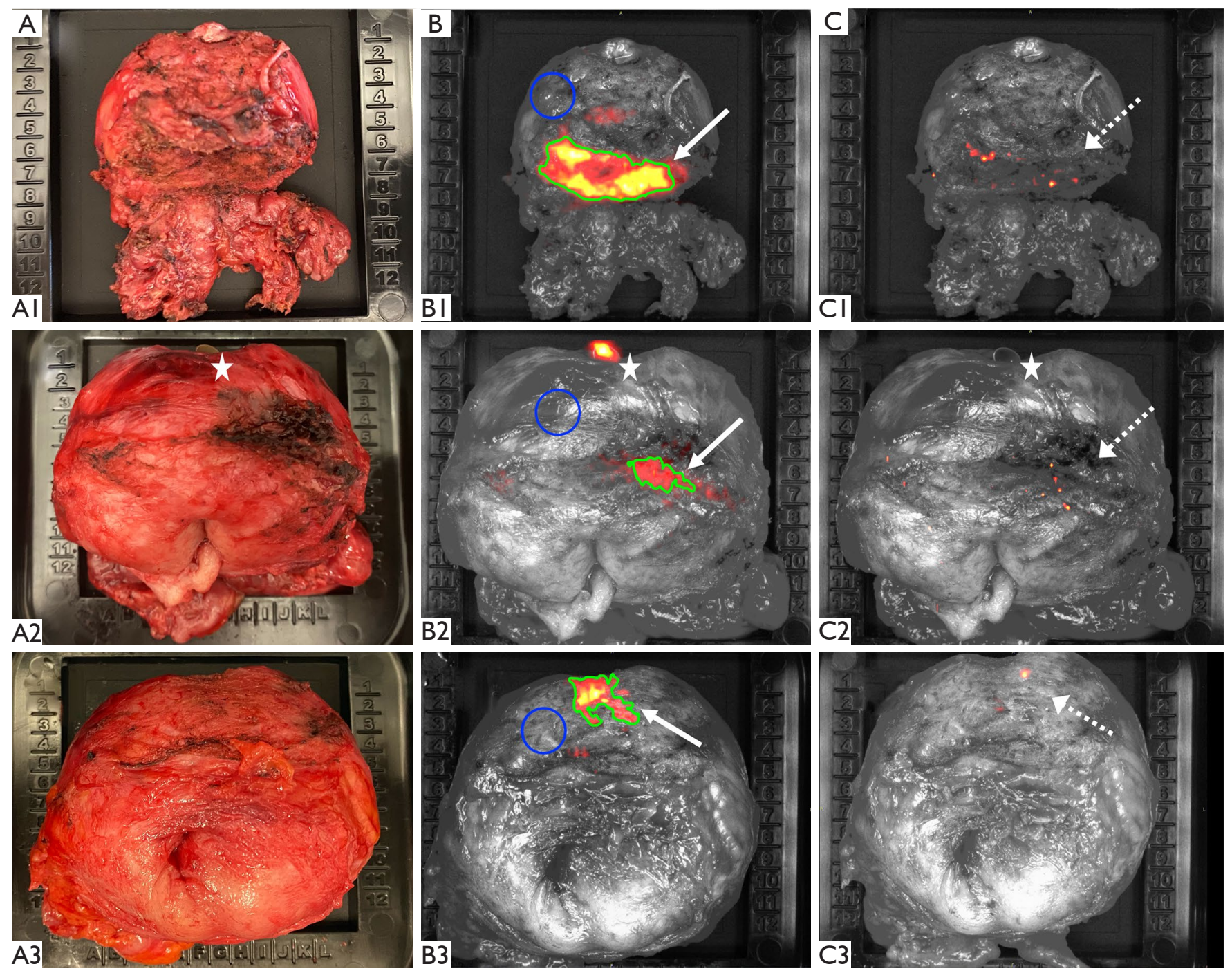

Figure 3 Anatomical (A) and grey-scale photographic images overlaid with Cerenkov signals, without the 550-nm optical short-pass filter (B) and with the 550-nm optical short-pass filter (C). All images show patients of the control group. The blue circles are background ROIs. Areas of suspicious CLI-intensity are labelled with green ROIs. (A1,B1,C1) Prostatectomy specimen with a high CLI-intensity at the ventral bladder neck is seen and represented by an arrow (TBR: 7.32, CNR: 34.32), correlating with the usage of diathermy intraoperatively. After application of the 550-nm optical short-pass filter these signals are close the background intensity level. The location of the area is marked here with a dotted arrow (TBR: 1.10, CNR: 3.84). (A2,B2,C2) Prostatectomy specimen with a moderate CLI-intensity at the bladder neck is seen and represented by an arrow (TBR: 3.43, CNR: 15.4), also correlating with the usage of diathermy intraoperatively. After application of the 550-nm optical short-pass filter these signals are similar to that of the background. The location of the area is marked here with a dotted arrow (TBR: 0.99, CNR: -0.03). The star marks a foreign body (glove residue). (A3,B3,C3) Prostatectomy specimen with a moderate CLI-intensity at the ventro-apical side of the prostate is seen and represented by an arrow (TBR: 3.99, CNR: 16.7), also correlating with the usage of diathermy intraoperatively. After application of the 550-nm optical short-pass filter these signals are close the background intensity level. The location of the area is marked here with a dotted arrow (TBR: 1.38, CNR: 2.64). ROIs, regions of interest; CLI, Cerenkov luminescence imaging; TBR, tumor to background ratio; CNR, contrast to noise ratio. 
Table 2 Cerenkov luminescence imaging measurements of each prostatectomy specimen

\begin{tabular}{|c|c|c|c|c|c|c|}
\hline Patient number & \multicolumn{3}{|c|}{ Without 550-nm OF } & \multicolumn{3}{|c|}{ With 550-nm OF } \\
\hline \multicolumn{7}{|l|}{ Study group } \\
\hline 1 & 3.36 & 11.44 & 1.80 & 1.10 & 0.54 & 0 \\
\hline 2 & 2.81 & 9.48 & 2.00 & 1.08 & 0.48 & 0.56 \\
\hline 4 & $5.73^{\star}$ & $14.98^{*}$ & $0.70^{\star}$ & $2.15^{\star}$ & $6.71^{*}$ & $0.20^{\star}$ \\
\hline 5 & 3.85 & 10.87 & 3.00 & 1.58 & 4.58 & 0.50 \\
\hline 6 & $3.33^{*}$ & $16.20^{*}$ & $0.75^{\star}$ & $2.10^{\star}$ & $6.54^{*}$ & $0.10^{*}$ \\
\hline 7 & $2.79^{\star}$ & $11.73^{*}$ & $0.47^{\star}$ & $1.50^{\star}$ & $2.44^{*}$ & $0.33^{*}$ \\
\hline 9 & 3.43 & 15.40 & 0.60 & 0.99 & -0.03 & 0 \\
\hline 10 & 7.32 & 34.32 & 2.70 & 1.10 & 3.84 & 0 \\
\hline
\end{tabular}

*, specimens with positive surgical margins. Study group patient 1-7 and the control group patient 8-10. Measured intensities are stated as tumor to background ratio (TBR) and contrast to noise ratio (CNR). Measurements were either performed without a 550-nm optical short-pass filter or with a 550-nm optical short-pass filter. The area of regions of interests (ROls) is stated in $\mathrm{cm}^{2}$. CNR, contrast to noise ratio; OF, optical short-pass filter.

Table 3 Cerenkov Luminescence measurements of the study group $(n=7)$ grouped to positive surgical margins $(n=3)$ and negative surgical margins $(\mathrm{n}=4)$

\begin{tabular}{|c|c|c|c|}
\hline Data of the study group $(n=7)$ & CLI Data without 550-nm short-pass filter & CLI Data with 550-nm short-pass filter & $P$ value \\
\hline Median TBR without PSM (IQR) & $3.61(2.95 ; 11.77)$ & $1.11(1.09 ; 1.47)$ & Not significant \\
\hline
\end{tabular}

TBR and CNR are stated as median values (IQR). CLI, Cerenkov Luminescence Imaging; CNR, contrast to noise ratio; IQR, interquartile ranges; PSM, positive surgical margin; TBR, tumor to background ratio.

contamination (35). One method for minimising the risk of prostate surface contamination from urine is to rinse the prostate with sodium chloride prior to CLI image acquisition. In the present study, the urine of all patients was drained through a urethral catheter immediately at the beginning of the surgery and all prostates were wiped to clean blood and fluids before the examination.

Noteworthy, the suspicious CLI signals at the bladder neck could be measured over 2 hours with approximately the same intensity. Therefore, the hypothesis that contamination by a PET tracer with a half-life of about 60 minutes leads to these signals is unlikely (29).

Intraoperative qualitative assessment of CLI data may lead surgeons to resect non-cancerous tissues, especially if no OF is used. Thus, quantitative assessment must be established to standardize CLI for multicentre and multisurgeon validation trials. In two recently published studies, TBR measurements were used for quantitative assessment of CLI intensities $(27,28)$. Grootendorst et al. demonstrated the clinical feasibility and safety of CLI in a small number 
of breast cancer patients using ${ }^{18} \mathrm{~F}-\mathrm{FDG}$ with a mean TBR of 2.41 (range, 1.63-3.22) (25). In a prospective study of five primary PC patients undergoing RP Olde Heuvel et al. postulate a mean TBR $>2$ to sufficiently distinguish between cancerous and benign tissue (28). According to our findings, elevated TBR and CNR correlate significantly with PSM if CLI is measured using the 550-nm OF. In contrast neither elevated TBR nor CNR values without $550-\mathrm{nm}$ OF, correlated with the presence of cancer cells close to the surface in our cohort. Reassuringly, our data are in line with the findings of Olde Heuvel et al. regarding the TBR. All patients with PSM and an increased CLI signal in the incision of the index lesion presented a TBR $>2$ (Table 3). The CNR values of this patient group were $>6$, indicating that a cut-off to distinguish between false-positive and PCinduced signal increases should be in this range.

In one patient with PSM, slightly elevated CLI intensities were seen without a positive CLI signal of the incised index lesion. The true-positive superficial signals were located in the area of the expected index lesion, left apex of the prostate (Figure 1, A2, B2, C2). Decay corrected 68-Ga-PSMA activity for the time of analysis was $0.89 \mathrm{kBq} / \mathrm{mL}, 316$ minutes after tracer injection. In an experimental study the minimum detection limit was $1.2 \mathrm{kBq} / \mathrm{mL}$ for $68-\mathrm{Ga}-\mathrm{PSMA}$. Here, Eppendorf tubes were filled with different amounts of tracer activities and examined for the minimal activity seen on CLI (36). Therefore, CLI might be more sensitive than previously thought. However, it remains unclear why we did not detect a signal in the incised specimen. Generally, underestimation of CLI signals could arise from the fact that some positrons escape from the surface of the specimen before their energy enter the Cerenkov threshold, which is likely for high energy Ga-68 positrons (37). According to Ciarrocchi et al. Cerenkov radiation is emitted on a cone around the particle direction. The characteristic emission angle depends on the velocity of the charged particle. The particle slows down depending on the emitted angle. Due to the lack of energy, however, no luminous phenomenon can be detected at a certain point. Therefore, it can be assumed that false positive findings are not influenced by the radiation angle of the charged particles. In addition, an evaluation of the specimens is performed in different positions (38). Another patient with a decay corrected activity of $0.34 \mathrm{kBq} / \mathrm{mL}$, at the time of CLI analysis, demonstrated an elevated signal of the incised prostate gland with a TBR of 2.15. Contrary, no positive CLI signals of the incised index lesion were detectable in four patients with negative surgical margins and a decay-corrected activity at the time of CLI analysis below $1.2 \mathrm{kBq} / \mathrm{mL}$. This is certainly mainly due to the low activity, but still contradicts the findings above where two PSM were detected at very low levels. This observation implies that there must be several other factors besides calculated activity, which have an influence on tracer accumulation and thus the CLI signal. It is known that lowgrade cancers have a lower PSMA expression, resulting in a shorter period for positive CLI signals (39). In addition, other oncological features such as the size of the index lesion could also influence SI. Furthermore, heterogeneity of tracer activity in the prostate is a common finding which is in accordance with observations of different PSMA expression levels between patients, and even heterogeneity within the same prostatic lesion. Therefore, larger scale studies with standardized operating procedures are clearly needed to evaluate the potential and the field of application before widespread adoption of this promising technique. In the future, the immediate intraoperative evaluation of the entire prostate surface could reduce or replace a frozen section examination. This could save time and personnel resources.

Some limitations of our manuscript merit further discussion. First, this study addresses a small selective population since only patients with an elevated risk for PSM due to advanced cancer were selected. Therefore, these are not representative for all PC patients undergoing RP. Second, the administrated tracer activity varied between the patients as well as the time period from tracer injection to CLI. These challenges might be overcome in case of an intraoperative tracer injection to standardize these parameters. In our study setting, tracer injection was only allowed within the context of a PET/CT. This causes significant time delays until the entire prostate surface is examined for elevated CLI intensities. Last, in 4 patients with NSM, imaging of tumor tissue by CLI revealed no elevated signals. This may be due to several oncological characteristics as described above. In our opinion, however, this is mainly due to the short half-life of the tracer. Therefore, further studies with either an intraoperative tracer injection or a tracer with a longer half-life such as 18-F-PSMA are mandatory.

\section{Conclusions}

Intraoperative 68-Ga-PSMA CLI in PC is a tool that warrants further investigation to improve RP. CLI provides high-resolution information of the whole prostate gland 
that may allow surgeons to assess margin status with a good correlation to histopathologic examination. The intraoperative analysis of the entire prostate surface with CLI in combination with OF is a promising approach to reduce artificial signals. Nevertheless, further clinical studies are necessary to determine sensitivity and specificity.

\section{Acknowledgments}

Funding: This work was supported by the "Deutsche Forschungsgemeinschaft - DFG” (Project number: Projekt HA 5160/5-1).

\section{Footnote}

Provenance and Peer Review: This article was commissioned by the Guest Editor (Tilman Todenhöfer) for the series "Management of Advanced Genitourinary Malignancies" published in Translational Andrology and Urology. The article has undergone external peer review.

Reporting Checklist: The authors have completed the STROBE reporting checklist. Available at http://dx.doi. org/10.21037/tau-20-1141

Data Sharing Statement: Available at http://dx.doi. org/10.21037/tau-20-1141

Conflicts of Interest: All authors have completed the ICMJE uniform disclosure form (available at http://dx.doi. org/10.21037/tau-20-1141). The series "Management of Advanced Genitourinary Malignancies" was commissioned by the editorial office without any funding or sponsorship. Dr. CK reports personal fees from Bristol-Myer-Squibb, outside the submitted work; Dr. NNH reports personal fees from Boston Scientific, personal fees from Novartis, personal fees from Intuitive Surgical, personal fees from LightPoint Medical, outside the submitted work; Dr. HR reports personal fees from Roche, grants and personal fees from Bristol-Myers Squibb, personal fees from Philips, other from Bayer, outside the submitted work; Dr. WPF reports personal fees from RadioMedix, personal fees from Parexel, personal fees from Bayer, personal fees from BTG, personal fees from Endocyte, during the conduct of the study; Dr. KH reports personal fees from Bayer, personal fees and other from Sofie Biosciences, personal fees from SIRTEX, non-financial support from ABX, personal fees from Adacap, personal fees from Curium, personal fees from Endocyte, grants and personal fees from BTG, personal fees from IPSEN, personal fees from Siemens Healthineers, personal fees from GE Healthcare, personal fees from Amgen, personal fees from Novartis, personal fees from ymabs, outside the submitted work; Dr. Radtke reports personal fees from Invivo, personal fees from Uronav, personal fees from Bender group, personal fees from Becklemann and Partners, personal fees from Saegeling Medizintechnik, other from Advanced Accelerator Applications, other from Novartis, outside the submitted work; Dr. BAH reports personal fees from ABX, personal fees from Bayer, personal fees and non-financial support from Lightpoint medical, Inc., personal fees and non-financial support from Janssen R\&D, grants from German Research Foundation, during the conduct of the study; personal fees and non-financial support from Bayer, personal fees and nonfinancial support from BMS, personal fees and non-financial support from AstraZeneca, personal fees from Pfizer, outside the submitted work; Dr. ST reports personal fees and other from Ipsen, personal fees from Eisai, personal fees from Bayer, personal fees from Janssen, personal fees from Novartis, personal fees from Bristol-Myers-Squibb, personal fees from BrachySolutions, outside the submitted work. The authors have no other conflicts of interest to declare.

Etbical Statement: The authors are accountable for all aspects of the work in ensuring that questions related to the accuracy or integrity of any part of the work are appropriately investigated and resolved. The study was conducted in accordance with the Declaration of Helsinki (as revised in 2013) and after written informed consent of the patients. The study was approved by institutional ethics board of the University Duisburg-Essen (approval number 19-8749-BO).

Open Access Statement: This is an Open Access article distributed in accordance with the Creative Commons Attribution-NonCommercial-NoDerivs 4.0 International License (CC BY-NC-ND 4.0), which permits the noncommercial replication and distribution of the article with the strict proviso that no changes or edits are made and the original work is properly cited (including links to both the formal publication through the relevant DOI and the license). See: https://creativecommons.org/licenses/by-nc-nd/4.0/.

\section{References}

1. EAU Guidelines. Edn. presented at the EAU Annual 
Congress Amsterdam 2020. EAU Guidelines Office; 2020.

2. Morlacco A, Sharma V, Viers BR, et al. The Incremental Role of Magnetic Resonance Imaging for Prostate Cancer Staging before Radical Prostatectomy. Eur Urol 2017;71:701-4.

3. Somford DM, Hamoen EH, Fütterer JJ, et al. The predictive value of endorectal 3 Tesla multiparametric magnetic resonance imaging for extraprostatic extension in patients with low, intermediate and high risk prostate cancer. J Urol 2013;190:1728-34.

4. Gandaglia G, Ploussard G, Valerio M, et al. The Key Combined Value of Multiparametric Magnetic Resonance Imaging, and Magnetic Resonance Imaging-targeted and Concomitant Systematic Biopsies for the Prediction of Adverse Pathological Features in Prostate Cancer Patients Undergoing Radical Prostatectomy. Eur Urol 2020;77:733-41.

5. Radtke JP, Hadaschik BA, Wolf MB, et al. The Impact of Magnetic Resonance Imaging on Prediction of Extraprostatic Extension and Prostatectomy Outcome in Patients with Low-, Intermediate- and High-Risk Prostate Cancer: Try to Find a Standard. J Endourol 2015;29:1396-405.

6. Petralia G, Musi G, Padhani AR, et al. Robot-assisted radical prostatectomy: Multiparametric MR imagingdirected intraoperative frozen-section analysis to reduce the rate of positive surgical margins. Radiology 2015;274:434-44.

7. Schlomm T, Tennstedt P, Huxhold C, et al. Neurovascular structure-adjacent frozen-section examination (NeuroSAFE) increases nerve-sparing frequency and reduces positive surgical margins in open and robot-assisted laparoscopic radical prostatectomy: experience after 11,069 consecutive patients. Eur Urol 2012;62:333-40.

8. Nyarangi-Dix J, Wiesenfarth M, Bonekamp D, et al. Combined Clinical Parameters and Multiparametric Magnetic Resonance Imaging for the Prediction of Extraprostatic Disease-A Risk Model for Patient-tailored Risk Stratification When Planning Radical Prostatectomy. Eur Urol Focus 2020;6:1205-12.

9. Gillitzer R, Thuroff C, Fandel T, t al. Intraoperative peripheral frozen sections do not significantly affect prognosis after nerve-sparing radical prostatectomy for prostate cancer. BJU Int 2011;107:755-9.

10. Wright JL, Dalkin BL, True LD, et al. Positive surgical margins at radical prostatectomy predict prostate cancer specific mortality. J Urol 2010;183:2213-8.
11. Perera M, Papa N, Roberts M, et al. Gallium-68 Prostate-specific Membrane Antigen Positron Emission Tomography in Advanced Prostate Cancer-Updated Diagnostic Utility, Sensitivity, Specificity, and Distribution of Prostate-specific Membrane Antigen-avid Lesions: A Systematic Review and Meta-analysis. Eur Urol 2020;77:403-17.

12. Yaxley JW, Raveenthiran S, Nouhaud FX, et al. Risk of metastatic disease on (68) gallium-prostate-specific membrane antigen positron emission tomography/ computed tomography scan for primary staging of 1253 men at the diagnosis of prostate cancer. BJU Int 2019;124:401-7.

13. Koschel S, Murphy DG, Hofman MS, et al. The role of prostate-specific membrane antigen PET/computed tomography in primary staging of prostate cancer. Curr Opin Urol 2019;29:569-77.

14. Kalapara AA, Nzenza T, Pan HYC, et al. Detection and localisation of primary prostate cancer using 68 gallium prostate-specific membrane antigen positron emission tomography/computed tomography compared with multiparametric magnetic resonance imaging and radical prostatectomy specimen pathology. BJU Int 2020;126:83-90.

15. Schwarzenboeck SM, Rauscher I, Bluemel C, Fendler WP, Rowe SP, Pomper MG, et al. PSMA ligands for PET imaging of prostate cancer. J Nucl Med 2017;58:1545-52.

16. Morris MJ. editor. Impact of PSMA-targeted imaging with 18F-DCFPyL-PET/CT on clinical management of patients (pts) with biochemically recurrent (BCR) prostate cancer (PCa): Results from a phase III, prospective, multicenter study (CONDOR). ASCO Virtual Scientific Program: American Society of Clinical Oncology, 2020.

17. Kesch C, Vinsensia M, Radtke JP, et al. Intraindividual Comparison of (18)F-PSMA-1007 PET/CT, Multiparametric MRI, and Radical Prostatectomy Specimens in Patients with Primary Prostate Cancer: A Retrospective, Proof-of-Concept Study. J Nucl Med 2017;58:1805-10.

18. Giesel FL, Hadaschik B, Cardinale J, et al. F-18 labelled PSMA-1007: biodistribution, radiation dosimetry and histopathological validation of tumor lesions in prostate cancer patients. Eur J Nucl Med Mol Imaging 2017;44:678-88.

19. Herlemann A, Wenter V, Kretschmer A, et al. (68)GaPSMA Positron Emission Tomography/Computed Tomography Provides Accurate Staging of Lymph Node Regions Prior to Lymph Node Dissection in Patients with 
Prostate Cancer. Eur Urol 2016;70:553-7.

20. van Kalmthout LWM, van Melick HHE, Lavalaye J, et al. Prospective Validation of Gallium-68 Prostate Specific Membrane Antigen-Positron Emission Tomography/ Computerized Tomography for Primary Staging of Prostate Cancer. J Urol 2020;203:537-45.

21. Hofman MS, Lawrentschuk N, Francis RJ, et al. Prostatespecific membrane antigen PET-CT in patients with high-risk prostate cancer before curative-intent surgery or radiotherapy (proPSMA): a prospective, randomised, multicentre study. Lancet 2020;395:1208-16.

22. Maurer T, Weirich G, Schottelius M, et al. Prostate-specific membrane antigen-radioguided surgery for metastatic lymph nodes in prostate cancer. Eur Urol 2015;68:530-4.

23. Das S, Thorek DL, Grimm J. Cerenkov imaging. Adv Cancer Res 2014;124:213-34.

24. Grootendorst MR, Cariati M, Kothari A, et al. Cerenkov luminescence imaging (CLI) for image-guided cancer surgery. Clin Transl Imaging 2016;4:353-66.

25. Grootendorst MR, Cariati M, Pinder SE, et al.

Intraoperative Assessment of Tumor Resection Margins in Breast-Conserving Surgery Using (18)F-FDG Cerenkov Luminescence Imaging: A First-in-Human Feasibility Study. J Nucl Med 2017;58:891-8.

26. Chin PT, Welling MM, Meskers SC, et al. Optical imaging as an expansion of nuclear medicine: Cerenkovbased luminescence vs fluorescence-based luminescence. Eur J Nucl Med Mol Imaging 2013;40:1283-91.

27. Darr C, Harke NN, Radtke JP, et al. Intraoperative 68GaPSMA Cerenkov Luminescence Imaging for Surgical Margins in Radical Prostatectomy: A Feasibility Study. J Nucl Med 2020;61:1500-6.

28. Olde Heuvel J, de Wit-van der Veen BJ, van der Poel HG, et al. 68Ga-PSMA Cerenkov luminescence imaging in primary prostate cancer: first-in-man series. Eur J Nucl Med Mol Imaging 2020;47:2624-32.

29. Fendler WP, Eiber M, Beheshti M, et al. (68)Ga-PSMA PET/CT: Joint EANM and SNMMI procedure guideline for prostate cancer imaging: version 1.0. Eur J Nucl Med Mol Imaging 2017;44:1014-24.

30. Ciarrocchi E, Vanhove C, Descamps B, et al. Performance evaluation of the LightPath imaging system for intraoperative Cerenkov luminescence imaging. Phys Med 2018;52:122-8.

31. Klein JS, Mitchell GS, Cherry SR. Quantitative assessment of Cerenkov luminescence for radioguided brain tumor resection surgery. Phys Med Biol 2017;62:4183-201.
32. Leitlinienprogramm Onkologie (Deutsche Krebsgesellschaft, Deutsche Krebshilfe, AWMF): Interdisziplinäre Leitlinie der Qualität S3 zur Früherkennung, Diagnose und Therapie der verschiedenen Stadien des Prostatakarzinoms, Langversion 5.1, 2019, AWMF Registernummer: 043/022OL. Available online: http://www.leitlinienprogramm-onkolo-gie.de/leitlinien/ prostatakarzinom/

33. Epstein JI, Egevad L, Amin MB, et al. The 2014 International Society of Urological Pathology (ISUP) Consensus Conference on Gleason Grading of Prostatic Carcinoma: Definition of Grading Patterns and Proposal for a New Grading System. Am J Surg Pathol 2016;40:244-52.

34. Darr C, Krafft U, Fendler WP, et al. First-in-man intraoperative Cerenkov luminescence imaging for oligometastatic prostate cancer using 68Ga-PSMA-11. Eur J Nucl Med Mol Imaging 2020;47:3194-5.

35. Budäus L, Leyh-Bannurah SR, Salomon G, et al. Initial Experience of (68)Ga-PSMA PET/CT Imaging in High-risk Prostate Cancer Patients Prior to Radical Prostatectomy. Eur Urol 2016;69:393-6.

36. Olde Heuvel J, de Wit-van der Veen BJ, Vyas KN, et al. Performance evaluation of Cerenkov luminescence imaging: a comparison of 68Ga with 18F. EJNMMI Phys 2019;6:17.

37. Mitchell GS, Gill RK, Boucher DL, et al. In vivo Cerenkov luminescence imaging: a new tool for molecular imaging. Philos Trans A Math Phys Eng Sci 2011;369:4605-19.

38. Ciarrocchi E, Belcari N. Cerenkov luminescence imaging: physics principles and potential applications in biomedical sciences. EJNMMI Phys 2017;4:14.

39. Hupe MC, Philippi C, Roth D, et al. Expression of Prostate-Specific Membrane Antigen (PSMA) on Biopsies Is an Independent Risk Stratifier of Prostate Cancer Patients at Time of Initial Diagnosis. Front Oncol 2018;8:623.

Cite this article as: Darr C, Fragoso Costa P, Kesch C, Krafft U, Püllen L, Harke NN, Hess J, Szarvas T, Haubold J, Reis H, Fendler WP, Herrmann K, Radtke JP, Hadaschik BA, Tschirdewahn S. Prostate specific membrane antigenradio guided surgery using Cerenkov luminescence imagingutilization of a short-pass filter to reduce technical pitfalls Transl Androl Urol 2021;10(10):3972-3985. doi: 10.21037/ tau-20-1141 